\title{
Epifluorescence Imaging of Electrochemically Switchable Langmuir-Blodgett Films of Nafion
}

\author{
Ligia Maria Moretto, ${ }^{\dagger}$ Thiago Kohls, ${ }^{\dagger}$ Arnaud Chovin, ${ }^{*}$ Neso Sojic, ${ }^{*, *}$ and Paolo Ugo ${ }^{* \dagger}$ \\ Department of Physical Chemistry, University of Venice, Santa Marta 2137, 30123 - Venice, Italy, and \\ Groupe NanoSystèmes Analytiques, Institut des Sciences Moléculaires, UMR 5255 CNRS, Université \\ Bordeaux 1, ENSCPB, France
}

Received December 21, 2007. Revised Manuscript Received February 26, 2008

\begin{abstract}
A combination of electrochemistry and luminescence methods was exploited to obtain information on the electrochemical activity and homogeneity of Nafion Langmuir-Blodgett films. The redox behavior of the Ru(bpy $)_{3}{ }^{2+}$ probe incorporated in the Nafion film was monitored by epifluorescence microscopy. The photoluminescent images, recorded by a charge-coupled device (CCD) camera, reflect the distribution of the probe in the film, which resulted as very uniform, particularly in comparison with spin-coated films. Apparent diffusion coefficients $\left(D_{\text {app }}\right)$ determined by cyclic voltammetry for films of less than 10 layers are in the range of $1 \times 10^{-12}$ to $8 \times 10^{-12} \mathrm{~cm}^{2} \mathrm{~s}^{-1}$, that is, 2 orders of magnitude lower than values reported in the literature for spin-coated Nafion films. The application to the electrode of a potential able to oxidize the luminescent $\mathrm{Ru}(\mathrm{bpy})_{3}{ }^{2+}$ to the nonluminescent $\mathrm{Ru}(\mathrm{bpy})_{3}{ }^{3+}$ switched off the photoluminescence with a response time that for the LB films was much shorter than that for the spin-coated ones. Experimental evidence and calculations indicate that lowering of the film thickness down to the nanometric level is very effective in shortening the switching time, notwithstanding the lowering of the $D_{\text {app }}$ value in LB films.
\end{abstract}

\section{Introduction}

Membranes of perfluorinated ionomers, such as Nafion, present unique properties as far as chemical, thermal, and mechanical stability, ion and water transport, and electrochemical characteristics are concerned. ${ }^{1}$ Nafion can be used not only as a preformed thick membrane but also in the form of hydroalcoholic dispersions, suitable for recasting thin films of the ionomer on metal surfaces. ${ }^{2,3}$ This gave rise to a simple and practical procedure to prepare polymer coated electrodes which display interesting characteristics for many analytical applications. ${ }^{4-6}$ The ability of Nafion films to preconcentrate redox cations, to reject anionic interferences, and to protect electrode surfaces from undesired adsorption and poisoning constitutes the basis for an electroanalytical technique named ion-exchange voltammetry. ${ }^{4,7}$

Recently, the interest in recasted Nafion extended to fuel cells, where it is used as an important component of the catalyst layer ${ }^{8}$ or, after dispersion with proper inorganic additives (montmorillonite, $\mathrm{Zr}$ phosphate, $\mathrm{Ti}, \mathrm{Pt}$, or $\mathrm{Si}$ oxides), to suppress fuel crossover in direct methanol fuel cells. ${ }^{9-11}$

* To whom correspondence should be addressed.E-mail: sojic@enscpb.fr (N.S.); ugo@unive.it (P.U.).

${ }^{\dagger}$ University of Venice.

* Université Bordeaux 1.

(1) Mauritz, K. A.; Moore, R. B. Chem. Rev. 2004, 104, 4535-4585.

(2) Rubinstein, I.; Bard, A. J. J. Am. Chem. Soc. 1981, 103, 5007-5013.

(3) Eisenberg, A.; Yeager, H. L. Perfluorinated Ionomer Membranes; ACS Symposium Series 180; American Chemical Society: Washington, DC, 1982.

(4) Ugo, P.; Moretto, L. M. Electroanalysis 1995, 7, 1105-1113.

(5) Ugo, P. In Encyclopedia of Sensors; Grimes, C. A., Dickey, E. C. , Pishko, M. V., Eds.; American Scientific Publishers: Stevenson Ranch, CA, 2006; Vol. $8, \mathrm{p} 67$.

(6) Ugo, P.; Moretto, L. M.; Vezzà, F. ChemPhysChem 2002, 3, 917-925. (7) Espenscheid, M. W.; Ghatak-Roy, A. R.; Moore, R. B.; Penner, R. M. Szentirmay, M. N.; Martin, C. R. J. Chem. Soc., Faraday Trans. 1 1986, 82, 1051-1070.

(8) Siroma, Z.; Fujiwara, N.; Ioroi, T.; Yamazaki, S.; Yasuda, K.; Miyazaki, Y. J. Power Sources 2004, 126, 41-45.

(9) Silva, R. F.; De Francesco, M.; Pozio, A. Electrochim. Acta 2004, 49, 3211-3219.

(10) Yang, B.; Manthiram, A. Electrochem. Commun. 2004, 6, 231-236.

(11) Lin, J.; Wycisk, R.; Pintauro, P. N.; Kellner, M. Electrochem. Solid-State Lett. 2007, 10, B19.
The recasting of Nafion on electrode surfaces is typically performed by microvolume evaporation or spin-coating. ${ }^{12}$ Notwithstanding the easiness of such coating procedures, it is widely demonstrated that films of recasted Nafion show properties strongly dependent on different parameters such as solvent choice, ${ }^{13,14}$ curing temperature, ${ }^{13-15}$ or relative humidity ${ }^{16}$ during the drying step. Lack of full control on such parameters can cause, sometimes, unsatisfactory reproducibility in the behavior of the coated electrode.

A better understanding of the properties and behavior of recasted Nafion can come from the possibility to build molecularly engineered films of the ionomer. ${ }^{17}$ Langmuir-Blodgett (LB) techniques received great attention thanks to the capability to build ultrathin films made of monolayers or multilayers ordered at a molecular level. ${ }^{18,19}$ This is particularly interesting for tailoring surface properties and controlling electron transfer processes at the electrode/solution interface. Recent results ${ }^{17,20,21}$ showed the feasibility of such an approach which allows the preparation of ultrathin (thickness as small as $2 \mathrm{~nm}^{20}$ ) films of Nafion and other ionomers. ${ }^{17}$ It was shown that Langmuir films of ionomers can be transferred on electrode surfaces by using Langmuir-Schaefer (LS) $)^{20,21}$ and Langmuir-Blodgett (LB) deposition techniques. ${ }^{17}$

While keeping the ion-exchange preconcentration capability and permselectivity typical of recasted ionomers, mass and charge

(12) Murray, R. W. In Electroanalytical Chemistry; Bard, A. J. , Ed.; Marcel Dekker: New York, 1984; Vol. 13, p 191.

(13) Weber, J.; Panda, P.; Kavan, L.; Jegorov, A. J. Electroanal. Chem. 1986, 200, 379-381.

(14) Moore, R. B., III; Martin, C. R. Macromolecules 1988, 21, 1334-1339.

(15) Hoyer, B.; Jensen, N. Talanta 1994, 41, 449-453.

(16) Striebel, K. A.; Scherer, G. G.; Haas, O. J. Electroanal. Chem. 1991, 304 , 289-296.

(17) Ugo, P.; Bertoncello, P.; Vezzà, F. Electrochim. Acta 2004, 49, 37853793.

(18) Roberts, G. G. Langmuir-Blodgett films; Plenum Press: New York, 1990. (19) Ulman, A. An introduction to ultrathin organic films from LangmuirBlodgett to self-assembly; Academic Press: London, 1991.

(20) Bertoncello, P.; Ram, M. K.; Notargiacomo, A.; Ugo, P.; Nicolini, C. Phys. Chem. Chem. Phys. 2002, 4, 4036-4043.

(21) Bertoncello, P.; Ugo, P. J. Braz. Chem. Soc. 2003, 14, 517-522. 


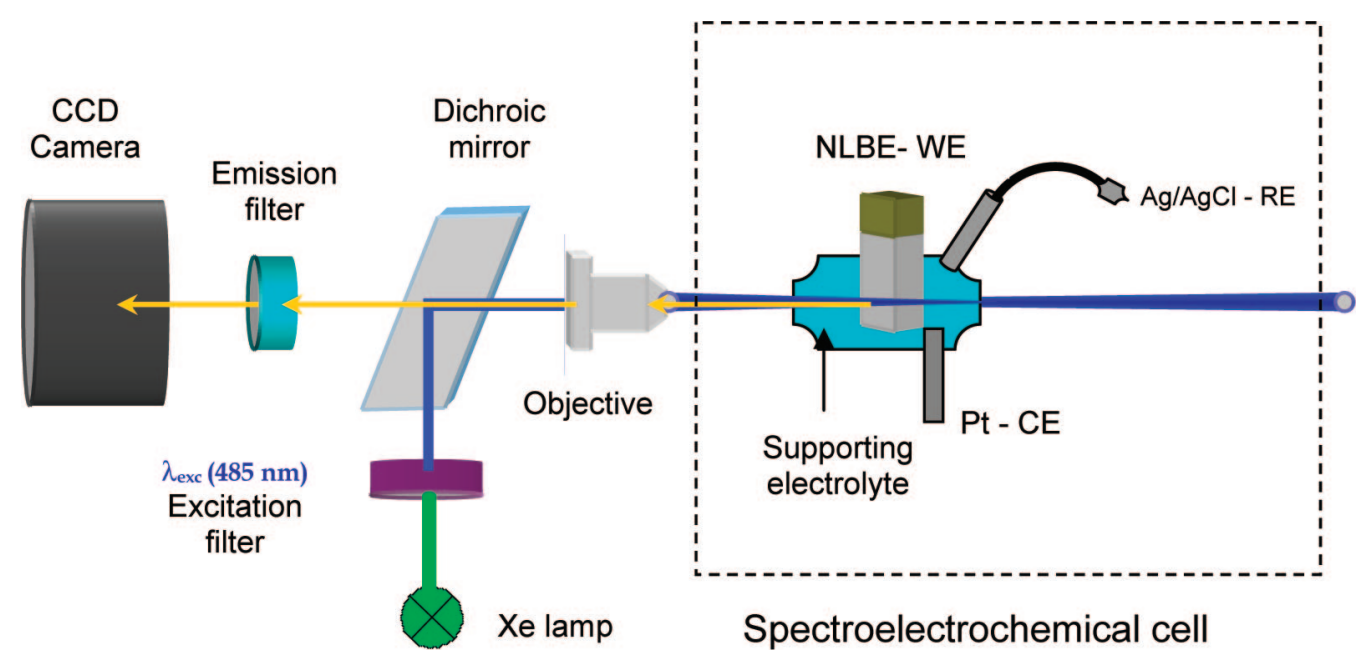

Figure 1. Schematic view of the experimental setup for electrochemical-epifluorescence measurements.

transfer processes relevant to electroactive species incorporated in LS and LB films of Nafion displayed some peculiarities with respect to "conventional" recasted films. ${ }^{21}$ Apparent diffusion coefficients of electroactive counterions are up to $2-3$ orders of magnitude lower than those in recasted coatings. ${ }^{22}$ In principle, this behavior can be attributed to a denser structure of the film, with multilayering of hydrophobic and hydrophilic domains as a consequence of the 2D ordering of the interfacial film during Langmuir compression in the trough. ${ }^{22}$ Very recently, Bertoncello et al. ${ }^{23,24}$ prepared LS films of Nafion and $\mathrm{Ru}(\mathrm{bpy}) 3_{3}{ }^{2+}$, forming a Langmuir monolayer with the mixture, which was transferred to the indium tin oxide (ITO) surface, in a procedure named by the authors as "one step". However, there is no clear experimental evidence to support whether LS- or LB-Nafion coatings retain the same clustered structure organized in hydrophobic/hydrophilic domains typical of "classical" Nafion (both as thick membranes or recasted films) or whether the $2 \mathrm{D}$ ordering created in the Langmuir interfacial film reflects in a sort of a layer-by-layer structuring where layers of sulfonic groups are sandwiched between layers of perfluorinated hydrophobic chains.

In order to gain deeper insight into this problem, it is necessary to complement the already very powerful electrochemical studies with additional information that could be gained with orthogonal techniques. For example, the cross-correlation of electrochemistry and luminescence methods (e.g., fluorescence, phosphorescence, electrochemiluminescence) is a very fruitful approach. Since the preliminary experiments by Allen and Patterson, ${ }^{25,26}$ the capability of the application of different forms of luminescence for understanding the structure, morphology, and behavior of materials $^{26,27}$ and films ${ }^{28-31}$ such as phospholipids ${ }^{32}$ as well as

(22) Bertoncello, P.; Ciani, I.; Li, F.; Unwin, P. R. Langmuir 2006, 22, 1038010388 .

(23) Bertoncello, P.; Wilson, N. R.; Unwin, P. R. Soft Matter 2007, 3, 13001307.

(24) Bertoncello, P.; Dennany, L.; Forster, R. J.; Unwin, P. R. Anal. Chem. 2007, 79, 7549-7553.

(25) Patterson, M. L. Raman Spectrometric and Fluorimetric Investigations of the surface and near-surface chemistry. Ph.D. Thesis, Indiana University, 1984.

(26) Patterson, M. L.; Allen, C. S. Anal. Chem. 1985, 57, 2751-2752.

(27) Pantano, P.; Kuhr, W. G. Anal. Chem. 1991, 63, 1413-1418.

(28) Buttry, D. A.; Anson, F. C. J. Am. Chem. Soc. 1982, 104, 4824-4829.

(29) Szentirmay, M. N.; Prieto, N. E.; Martin, C. R. J. Phys. Chem. 1985, 89, 3017-3023.

(30) Zhang, J.; Zhao, F.; Kaneko, M. Electrochim. Acta 1999, 44, 3367-3375.

(31) Shiroishi, H.; Kazunori Ishikawa, K.; Kaneko, M. J. Electroanal. Chem. 2002, 536, 145-150.

(32) Biswas, N.; Shanmukh, S.; Waring, A. L.; Walther, F.; Wang, Z.; Chang, Y.; Notter, R. H.; Dluhy, R. A. Biophys. Chem. 2005, 113, 223-232. for the study of the molecular dynamics of biological particles ${ }^{33}$ is well-documented in the literature. The progressive development of a sensitive charge-coupled device (CCD) camera added spatial and temporal resolution to visualize luminescence phenomena which are electrochemically modulated (e.g., imaging the functional area of an electrode). Indeed, the local variations of the luminescence intensity provide a global and dynamic picture of the analyzed microenvironment, which is not accessible otherwise by simple current measurements. The tris $\left(2,2^{\prime}-\right.$ bipyridine)ruthenium(II) complex is a model redox photoluminescent probe because of its extraordinary combined electrochemical, photophysical, and photochemical properties. This transition metal complex shows strong luminescence in solution at room temperature. When this complex is reversibly oxidized at the electrode surface, its luminescent properties change completely: the $\mathrm{Ru}(\mathrm{II})$ state is photoluminescent $\left(\lambda_{\mathrm{exc}}=485 \mathrm{~nm}\right.$; $\lambda_{\mathrm{em}}=605 \mathrm{~nm}$ ), whereas the $\mathrm{Ru}(\mathrm{III})$ state is not. For example, such a characteristic has been exploited by Kuhr et al. to image the spatial segregation of enzymes on carbon fiber microelectrodes. ${ }^{34}$ Weber et al. reported also a novel chromatographic detection method using this complex as a postcolumn fluorogenic reagent. ${ }^{35}$ Heineman et al. presented an original spectroelectrochemical detection scheme that incorporates multiple internal reflection spectroscopy at an optically transparent electrode coated with a selective Nafion film loaded with $\mathrm{Ru}(\mathrm{bpy})_{3}{ }^{2+} \cdot{ }^{36}$ More recently, Amatore et al. monitored the real-time dynamic profiling of concentration gradients during electrochemical experiments using $\mathrm{Ru}(\mathrm{bpy})_{3}{ }^{2+} .37$

The present research aims to establish the basis for exploiting a similar approach to monitor by epifluorescence microscopy and in situ electrochemistry the redox behavior of the $\mathrm{Ru}(\mathrm{bpy})_{3}{ }^{2+}$ probe incorporated into the LB-Nafion film. This requires a detailed study on the factors ruling the electrochemical and epifluorescence behavior of the $\mathrm{Ru}(\mathrm{bpy}) 3^{2+}$ complex incorporated in the LB film. Using our approach, the photoluminescent images reflect the local concentration of the $\mathrm{Ru}(\mathrm{bpy}) 3^{2+}$ complex immobilized in the LB-Nafion multilayer structure. The study

(33) Yang, W.; Musser, S. M. Methods 2006, 39, 316-328.

(34) Rosenwald, S. E.; Dontha, N.; Kuhr, W. G. Anal. Chem. 1998, 70, $1133-$ 1140.

(35) Woltman, S. J.; Even, W. R.; Weber, S. G. Anal. Chem. 1999, 71, 15041512 .

(36) Kaval, N.; Seliskar, C. J.; Heineman, W. R. Anal. Chem. 2003, 75, 63346340 .

(37) Amatore, C.; Chovin, A.; Garrigue, P.; Servant, L.; Sojic, N.; Szunerits, S.; Thouin, L. Anal. Chem. 2004, 76, 7202-7210. 
of changes in photoluminescence after the application of a sufficiently high potential to oxidize luminescent $\mathrm{Ru}(\mathrm{II})$ to nonluminescent $\mathrm{Ru}(\mathrm{III})$ can give information on the response time and on the electroactivity of all the photoactive probes in the film. In other words, the electrochemical activity and homogeneity of the LB-Nafion film are directly imaged.

\section{Experimental Section}

2.1. Materials. Tris(2,2'-bipyridyl)ruthenium(II) chloride hexahydrate, potassium nitrate, phosphate buffered saline ( $\mathrm{PBS}, \mathrm{pH}=7.4$ ), sodium chloride, and Nafion 117 solution (equivalent weight (EW) $1117,5 \% \mathrm{w} / \mathrm{v}$ in a mixture of low molecular weight alcohol) were purchased from Sigma-Aldrich. Stock solutions of Nafion at the required concentration were prepared by proper dilution of the commercial solution with methanol. All solutions were prepared in $18 \mathrm{M} \Omega$ water (nanopure). Polished float glass, $\mathrm{SiO}_{2}$ passivated/ indium tin oxide (ITO), was from Delta Technologies $\left(R_{\mathrm{S}}=4-8\right.$ $\Omega)$.

2.2. Procedure and Instrumentation. LB Films. Interfacial films of Nafion were prepared using a Langmuir-Blodgett trough (Langmuir KSV 2000 trough, KSV Instruments Ltd., Finland). The surface pressure was measured by means of a Wilhelmy balance with an accuracy of $0.2 \mathrm{mN} \mathrm{m}^{-1}$. A volume of $300 \mu \mathrm{L}$ of Nafion $0.05 \%$ was spread as three separate aliquots of $100 \mu \mathrm{L}$. After each addition, the interfacial film was compressed and decompressed up to a final compression after the last addition. As suggested in the literature, this separate addition method allows better repeatability and higher compressibility of interfacial Langmuir films of polymers. ${ }^{18,38}$ On the basis of the shapes of relevant Langmuir isotherms, typical surface pressures for performing the LB-Nafion deposition were $20 \mathrm{mN} \mathrm{m}^{-1}$. ITO glass slides were cleaned in a heated ultrasonicated ethanolamine-water 1:4 solution and copiously rinsed with MilliQ water before the transfer of the LB-Nafion film. The thicknesses of the LB films were measured by AFM following the procedure reported in ref 20 . By this procedure, a dry thickness value is obtained; however, results of measurements carried out in solution indicated that swelling of the films is negligible. ${ }^{23}$

Nafion spin-coating was performed with a Servodyne mixer/ spinner comprising an inverted mixer head and a digital RPM controller (Cole-Parmer Instrument, Veron Hills, IL). The ITO electrode was mounted horizontally onto the spinner mixer head. An aliquot of $20 \mu \mathrm{L}$ of Nafion $0.05 \%$ in methanol was dispensed onto the ITO surface and was spun at $2000 \mathrm{rpm}$ for $1 \mathrm{~min}$. The Nafion layer was then dried in air at room temperature for $15 \mathrm{~min}$. The thickness of the coatings was measured with a Tencor $\alpha$-STEP 500 profilometer and was estimated to be $250 \pm 25 \mathrm{~nm}$.

Electrochemical and Epifluorescence Measurements. The electrochemical measurements were performed using a CHI model 660B voltammetric analyzer, controlled by its own software. A standard three electrode cell configuration was used. $\mathrm{Ag} / \mathrm{AgCl}$ (KCl-saturated) was used as the reference electrode, and all potentials were measured and reported with respect to this electrode. The counter electrode was a platinum coil. Unless otherwise indicated, the area of the ITO electrodes was $0.07 \mathrm{~cm}^{2}$.

Photoluminescence imaging was performed with a modified epifluorescence microscope (BX-30, Olympus) interfaced with a potentiostat PGSTAT 12 Autolab (EcoChemie) for the voltammetric measurements. The experimental assembly is shown in Figure 1. Light from a $75 \mathrm{~W}$ xenon arc lamp was optimized on the region of interest. Light was then collimated and passed through a $485 \pm 11$ $\mathrm{nm}$ excitation filter (Omega Optical) corresponding to the $\mathrm{Ru}$ (II) excitation wavelength. The selected wavelength was reflected at $90^{\circ}$ by a $540 \mathrm{~nm}$ dichroic mirror and focused with a $20 \times$ microscope objective. The sample, that is, the Nafion-coated ITO loaded with $\mathrm{Ru}(\mathrm{bpy})_{3}{ }^{2+}$, was located in a spectroelectrochemical cell, and the focal plane corresponded to the electrode surface. The photoluminescence from the electrode surface was filtered by a band-pass

(38) Gaines, G. L. Insoluble monolayers at liquid-gas interfaces; Interscience Publishers: New York, 1966. filter $(605 \pm 25 \mathrm{~nm})$ to ensure that only $\mathrm{Ru}$ (II) photoluminescence was observed. The photoluminescence images produced while changing the electrochemical potential applied to the sample were acquired by a CCD camera (Roper Scientific), which was fitted with a back-illuminated chip (Marconi 47-10) that has $1024 \times 1024$ pixels. Maximum pixel readout rate was $1 \mathrm{MHz}$ at 16 bits. The spatial resolution of the mapping is about $300 \mathrm{~nm}$. The camera head cools the chip thermoelectrically to $-40{ }^{\circ} \mathrm{C}$.

\section{Results and Discussion}

3.1. Cyclic Voltammetry of Ru(bpy $)^{2+}$ at LB-Nafion Films. Cyclic voltammetry was used for the study of the ion-exchange incorporation and electrochemical behavior of $\mathrm{Ru}(\mathrm{bpy}) 3^{2+}$ in an ITO electrode coated with Nafion-LB films. Figure 2 shows the voltammetric patterns recorded at a 9-layer Nafion-LB electrode (NLBE) dipped in $2 \times 10^{-5} \mathrm{M} \mathrm{Ru}(\mathrm{bpy})_{3}{ }^{2+}, 0.01 \mathrm{M} \mathrm{KNO}_{3}$ solution; the full line refers to the first scan recorded soon after dipping the coated electrode in the solution, while the dashed line voltammogram was recorded $3 \mathrm{~min}$ after. The cyclic voltammogram shown by the dotted line was recorded at a bare ITO in a $5 \times$ more concentrated $\mathrm{Ru}(\mathrm{bpy}) 3_{3}{ }^{2+}$ solution. Comparison of Figure 2 with literature data indicates that the process involved is the one electron reversible oxidation of $\mathrm{Ru}(\mathrm{bpy}) 3_{3}{ }^{2+}$ preconcentrated by ion-exchange within the Nafion-LB coating:

$$
\mathrm{Ru}(\text { bpy })_{3}{ }^{2+} \leftrightharpoons \mathrm{Ru}(\text { bpy })_{3}{ }^{3+}+\mathrm{e}^{-}
$$

Actually, the fact that reaction (1) occurs within an ion-exchange coating is responsible both for the positive shift in the $E_{1 / 2}$ values (calculated as $E_{1 / 2}=\left(E_{\mathrm{pf}}+E_{\mathrm{pb}}\right) / 2$ ) with respect to $E_{1 / 2}$ obtained at the bare ITO electrode ${ }^{4,39}$ and for the fact that this shift increases with increasing dipping time. Taking into account previous studies $^{40}$ for the case of $\mathrm{Os}(\mathrm{bpy})_{3}{ }^{2+}$ incorporated in recasted Nafion, such a shift in the $E_{1 / 2}$ values suggests a lowering of the hydration of the LB-Nafion film with increasing incorporation of the ruthenium complex. Even a partially dehydrated film contains less water, thus causing an enhancement of ion pairing between electroactive counterions and the sulfonic groups of Nafion. Consequently, electrochemical oxidation of the redox centers becomes more energy demanding so that $E_{1 / 2}$ shifts toward more positive potential values while increasing the $\mathrm{Ru}(\mathrm{bpy}) 3_{3}{ }^{2+}$ loading. The higher condensation degree of Nafion-LB films with respect to recasted ones is responsible for stronger interactions between neighboring sulfonic groups, with a consequent higher sensitivity of electrochemical responses to small changes in the hydration state of the ionomer film.

The time dependence of the increase in the oxidation peak current for the NLBE (Figure 3) shows that peak current increases approximately $50 \%$ only $1 \mathrm{~min}$ after dipping in the $\mathrm{Ru}(\mathrm{bpy})_{3}{ }^{2+}$ solution, rapidly reaching a plateau value after approximately 15 min. This evidence indicates a quick ion-exchange incorporation of $\mathrm{Ru}(\mathrm{bpy})_{3}{ }^{2+}$ within the ultrathin Nafion-LB film.

Figure 3 (squares) reports the time dependence of the decrease in the oxidation peak current recorded when the electrode is transferred from the $\mathrm{Ru}(\mathrm{bpy})_{3}{ }^{2+}$ solution to pure supporting electrolyte. This plot shows a decrease in the peak current (approximately 60\%) immediately after transfer, which stabilizes to 0.9 of the initial signal $1 \mathrm{~h}$ after transfer. This indicates that a significant amount of $\mathrm{Ru}(\mathrm{bpy}){ }_{3}{ }^{2+}$ remains strongly incorporated in the Nafion film after the transfer to pure supporting electrolyte solution. This is related to the fact that $\mathrm{Ru}(\mathrm{bpy}){ }_{3}{ }^{2+}$ interacts not only with the ion-exchange sites but also with the hydrophobic

(39) Naegeli, R.; Redepenning, J.; Anson, F. C. J. Phys. Chem. 1986, 90, $6227-6232$

(40) Martin, C. R.; Rubinstein, I.; Bard, A. J. J. Am. Chem. Soc. 1982, 104, 4817-4824. 


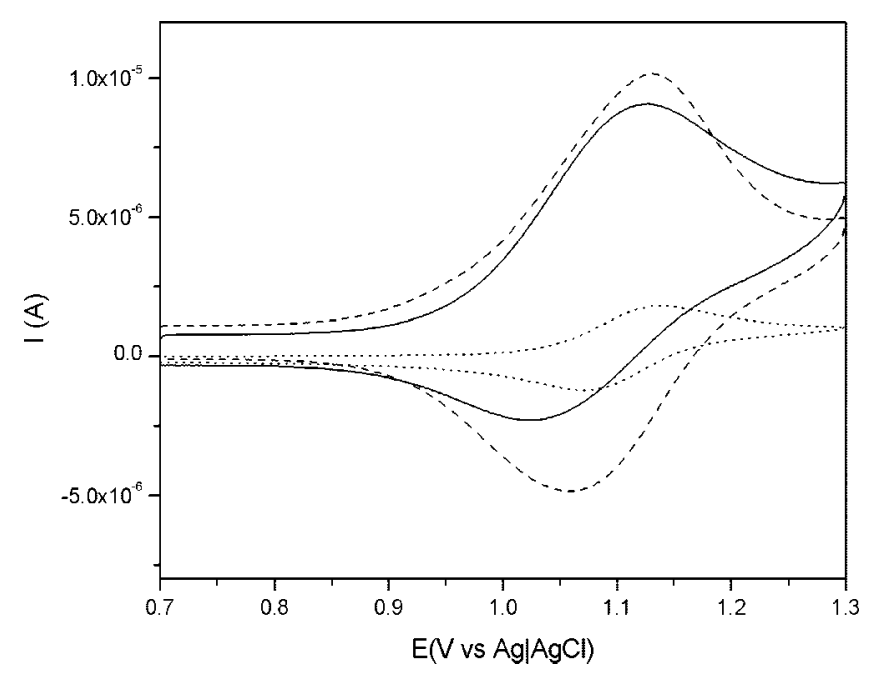

Figure 2. Cyclic voltammograms recorded at a 9-layer NLBE in $2 \times$ $10^{-5} \mathrm{M} \mathrm{Ru}(\mathrm{bpy})_{3}{ }^{2+}, 0.01 \mathrm{M} \mathrm{KNO}_{3}$ solution, first cycle (solid line), and after ion-exchange ( $3 \mathrm{~min}$ ) (dashed line) and at a bare ITO electrode in $1 \times 10^{-4} \mathrm{M} \mathrm{Ru}\left(\right.$ bpy) ${ }^{2+}$ (dotted line) (scan rate $50 \mathrm{mV} \mathrm{s}^{-1}$; electrode area $\left.0.07 \mathrm{~cm}^{2}\right)$.

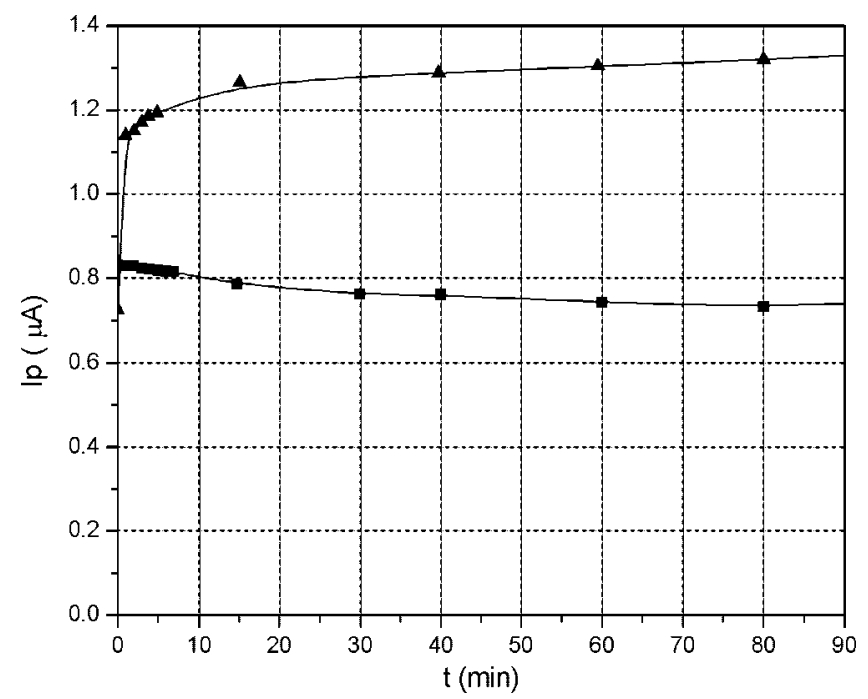

Figure 3. Dependence of the oxidation peak current on the incorporation time (triangles) and on the release time of the analyte in only supporting electrolyte (squares). Loading in $2 \times 10^{-6} \mathrm{M} \mathrm{Ru}(\text { bpy })_{3}{ }^{2+}, 0.01 \mathrm{M} \mathrm{KNO}_{3}$ solution; release in pure supporting electrolyte.

regions of Nafion, with the latter continuing to play a relevant role also in LB-Nafion films. ${ }^{29}$

The effect of the scan rate on the voltammetric behavior of $\mathrm{Ru}(\mathrm{bpy}){ }_{3}{ }^{2+}$ was studied as a function of the number of Nafion-LB layers. Figure 4 presents the dependence of peak current on the scan rate and on the square root of the scan rate for films with different numbers of LB layers, for NLBEs loaded in $2 \times 10^{-6}$ $\mathrm{MRu}(\mathrm{bpy}) 3_{3}{ }^{2+}$ and transferred to pure supporting electrolyte $(0.01$ $\mathrm{M} \mathrm{KNO}_{3}$ ). These plots show a linear behavior with the scan rate up to a maximum value that is $0.2,0.1$, and $0.05 \mathrm{~V} \mathrm{~s}^{-1}$ for 1 , 3 , and 9 layers, respectively. At higher scan rates, after a transition region, peak currents become linearly dependent on $v^{1 / 2}$. At the maximum scan rate where thin-layer behavior $\left(i_{\mathrm{p}} \alpha v\right)$ is observed, the thickness of the diffusion layer $\left(2 D_{\text {app }} t\right)^{1 / 2}$ is approximately equal to the polymer film thickness $\phi$, that is (a)
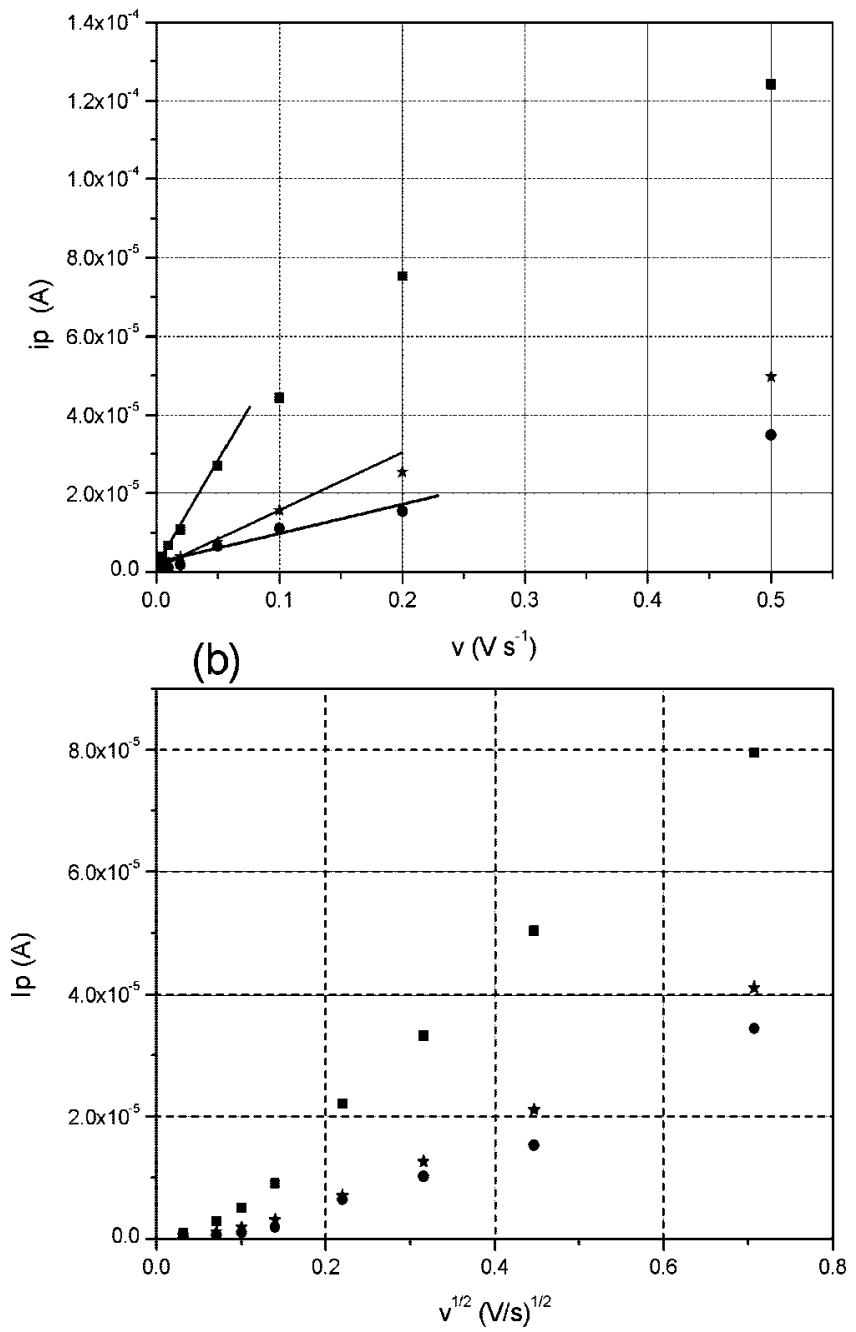

Figure 4. Variations of the oxidation peak current with the scan rate (a) and with the square root of the scan rate (b) for 1 (circles), 3 (stars) and 9 (squares) LB-Nafion layers.

$$
\phi=\left(2 D_{\text {app }} t\right)^{1 / 2}
$$

For shorter time scales, that is, for higher scan rates, the thickness of the diffusion layer becomes shorter than the thickness of the film, that is, $\left(2 D_{\text {app }} t\right)^{1 / 2} \ll \phi$. Under this condition, the peak current becomes a linear function of $v^{1 / 2}$. Note that even in the thinnest film (one layer) the thickness of the deposit is higher than the one expected for one flat monolayer of monomeric units but corresponds to approximately seven "monolayers", ${ }^{17}$ where,

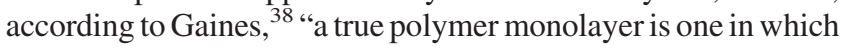
every monomer segment of each molecule is constrained to remain in the interface during the period of the experiment". This explains why a diffusion control on charge transfer is observed only at very short times ( $<100 \mathrm{~ms}$ for one LB layer).

The above-described results confirm that the transition between thin-layer-like behavior $\left(i_{\mathrm{p}} \alpha v\right)$ and diffusion controlled behavior $\left(i_{\mathrm{p}} \alpha v^{1 / 2}\right)$ is observed at progressively lower scan rates with an increasing number of Nafion-LB layers.

$D_{\text {app }}$ values can be calculated by eq 2 or by eq 3

$$
D_{\text {app }}=\left(S \phi / 2.69 \times 10^{5} \mathrm{~m}\right)^{2}
$$

where $m$ is number of moles of $\mathrm{Ru}(\mathrm{bpy})_{3}{ }^{2+}$ incorporated in the film, measured by integration of the relevant oxidation peak under thin layer conditions $\left(5 \mathrm{mV} \mathrm{s}^{-1}\right)$, and $S$ is the slope of the 

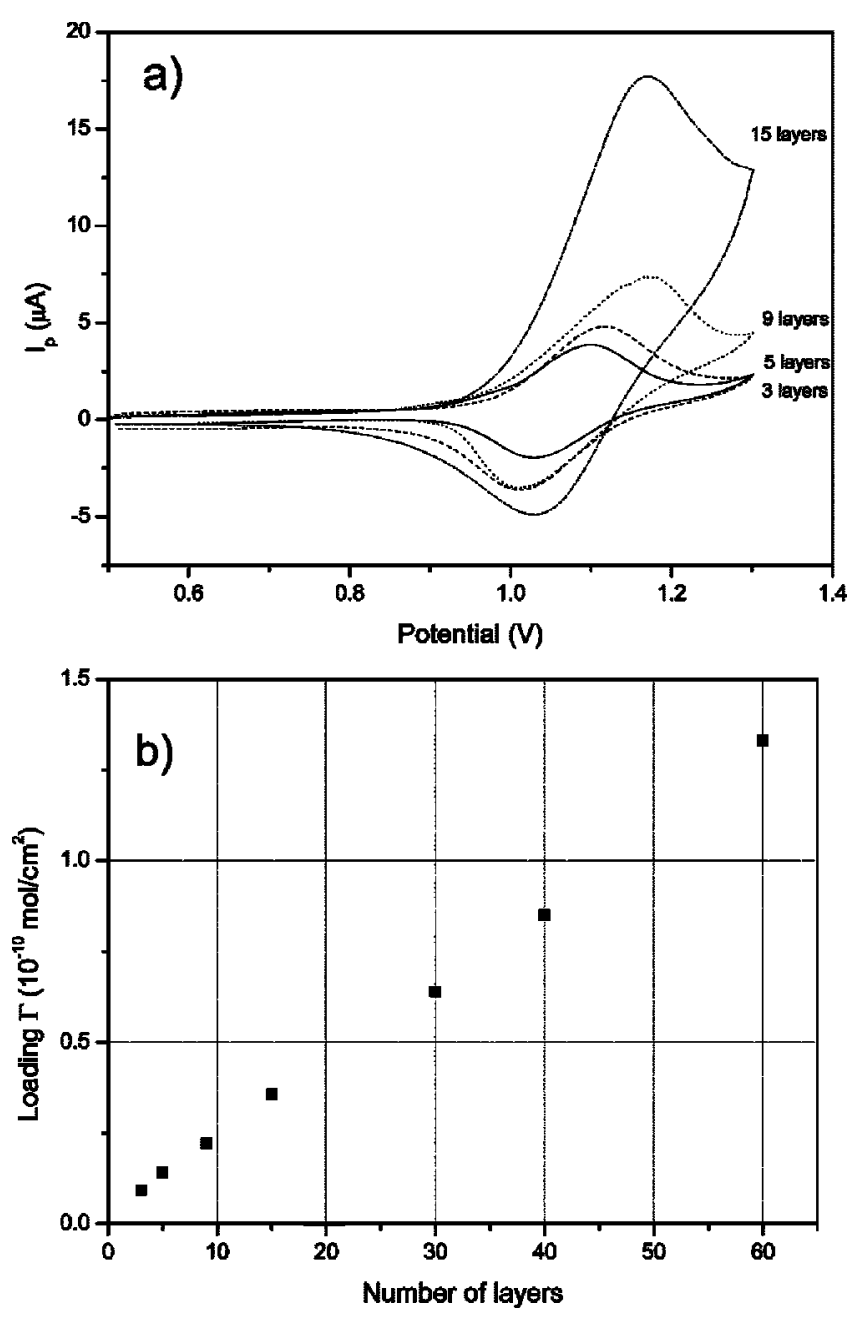

Figure 5. (a) Cyclic voltammograms recorded in $0.01 \mathrm{M}$ PBS solution after ion-exchange equilibrium in $1 \mathrm{mM} \mathrm{Ru}(\mathrm{bpy})_{3}{ }^{2+}$ at $3,5,9$, and 15 Nafion-LB layers deposited at an ITO electrode (scan rate $50 \mathrm{mV} \mathrm{s}^{-1}$; electrode area $0.90 \mathrm{~cm}^{2}$ ). (b) surface coverage of $\mathrm{Ru}(\mathrm{bpy})_{3}{ }^{2+}$ in the film as a function of the number of Nafion-LB layers calculated from the anodic peak area in the same experimental conditions as in (a).

linear portion of the plot $i_{\mathrm{p}} \alpha v^{1 / 2}$. Note that eq 3 is the corrected version of equation 1 in ref 21. $D_{\text {app }}$ values calculated by eqs 2 and 3 are listed in columns 6 and 7 in Table 1 . These values are in good agreement for NLBEs with 3 and 9 layers, while slightly less satisfactory agreement is obtained with 1 layer. Moreover, $D_{\text {app }}$ values increase slightly with increasing number of LB layers. $D_{\text {app }}$ values for $\mathrm{Ru}(\mathrm{bpy})_{3}{ }^{2+}$ in Nafion-LB coating measured by us are in agreement with the trend recently reported by Bertoncello et al. for Nafion-LS films. ${ }^{22}$ These values are approximately 2 orders of magnitude lower than the values measured in recasted films. ${ }^{40,42}$ The present data confirms that the $D_{\text {app }}$ values are typically lower in LB films of Nafion with respect to recasted coatings. Such a trend was observed also for other cations such as (ferrocenylmethyl)trimethylammonium $\left(\mathrm{FA}^{+}\right)$and $\mathrm{Ru}\left(\mathrm{NH}_{3}\right)_{6}{ }^{3+} \cdot 22$

By performing the loading in more concentrated $\mathrm{Ru}(\mathrm{bpy})_{3}{ }^{2+}$ solutions than those in Figure 2, the sulfonate sites of the NLBE are expected to be saturated by the electroactive complex. Figure 5 shows cyclic voltammograms recorded after saturation loading in $1 \mathrm{mM} \mathrm{Ru}(\mathrm{bpy})_{3}{ }^{2+}$ for NLBEs coated with different numbers

(41) Shi, M.; Anson, F. C. J. Electroanal. Chem. 1996, 415, 41-46. 2507.

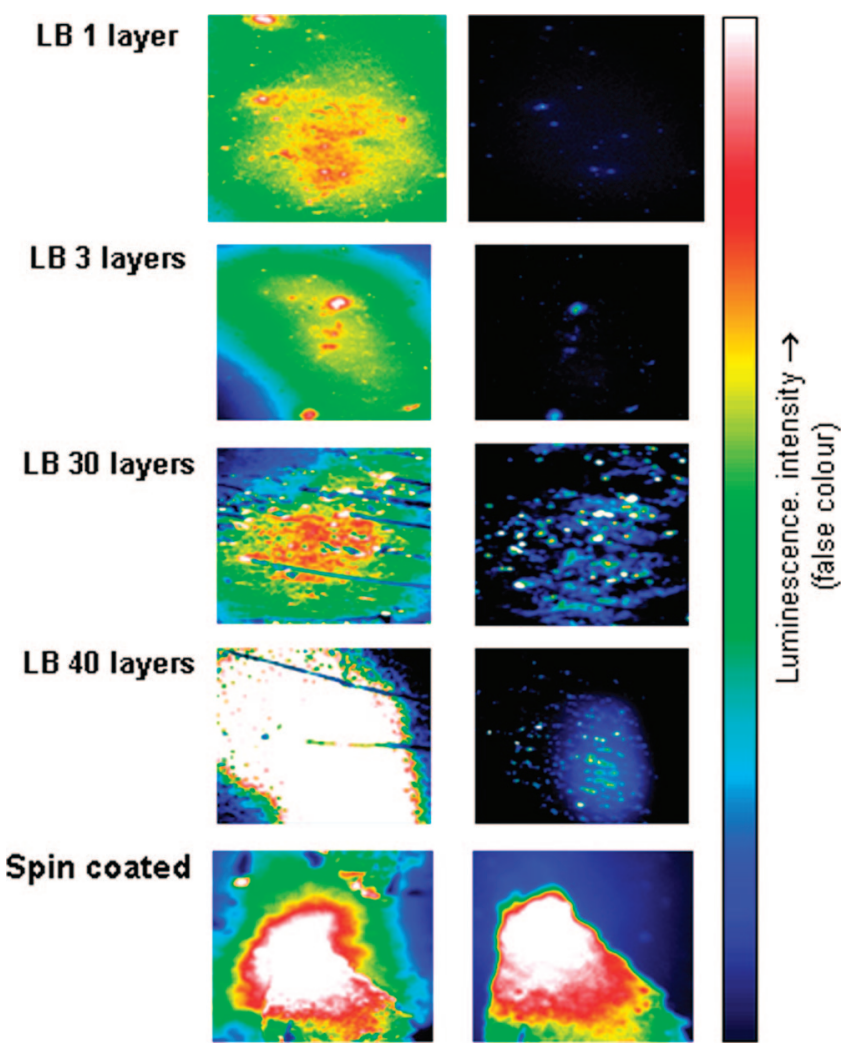

Figure 6. Epifluorescence images of LB-Nafion films with increasing number of layers (1, 3, 30, and 40) and spin-coated Nafion film before (left side) and after (right side) application of $1.2 \mathrm{~V}$. The right side images were acquired $1 \mathrm{~s}$ after the oxidizing potential step for the LB films and $20 \mathrm{~s}$ for the spin-coated Nafion film. The films were loaded in $10^{-3} \mathrm{M} \mathrm{Ru}(\mathrm{bpy})_{3}{ }^{2+}$. The size of the imaged area is $300 \times 300 \mu \mathrm{m}^{2}$.

of LB layers, namely 3, 5, 9, and 15 . Coulometric integration of the peak currents recorded at low scan rates $\left(5 \mathrm{mVs}^{-1}\right)$ allows one to calculate $\mathrm{Ru}(\mathrm{bpy})_{3}{ }^{2+}$ loadings. As shown in Figure $5 \mathrm{~b}$, they scale linearly with the number of LB layers up to, at least, 60 layers. From the slope of this plot, taking into account that two sulfonic groups interact with one $\mathrm{Ru}(\mathrm{bpy})_{3}{ }^{2+}$ ion, the surface coverage for one LB layer can be estimated as $4 \times 10^{-12}$ equiv $\mathrm{cm}^{-2}$. Such a voltammetric characterization of $\mathrm{Ru}(\mathrm{bpy})_{3}{ }^{2+}$ in the NLBE gave important information necessary for the following epifluorescence study.

3.2. Epifluorescence Imaging. The photoluminescence imaging of the film can give direct information on the morphology of the film, since the distribution of the light should reflect the local concentration of the $\mathrm{Ru}(\mathrm{bpy})_{3}{ }^{2+}$ complex immobilized in the LB-Nafion multilayer structure. Moreover, the study of the electrochemical switching of epifluorescence can furnish information on the response time and ratio between photoluminescence and electroactive probes in the film. $\mathrm{Ru}(\mathrm{bpy}){ }_{3}{ }^{2+}$ emits a strong luminescence with a maximum at $605 \mathrm{~nm}$, while the oxidized form is not photoluminescent. The experimental setup is such that both the excitation $(485 \mathrm{~nm})$ and the emitted radiation $(605$ $\mathrm{nm}$ ) pass through the objective of a microscope, with the former being focused on the surface of the modified electrode, using the setup shown in Figure 1.

Figure 6 shows typical photoluminescence images of Nafion films prepared by the LB technique and by spin-coating. The images on the left side were recorded when applying at the NLBE a potential of $0 \mathrm{~V}$, in which the $\mathrm{Ru}$ (II) complex is in the photoluminescent form; the images on the right side were recorded after applying an oxidizing potential of $1.2 \mathrm{~V}$, where $\mathrm{Ru}(\mathrm{III})$ is 

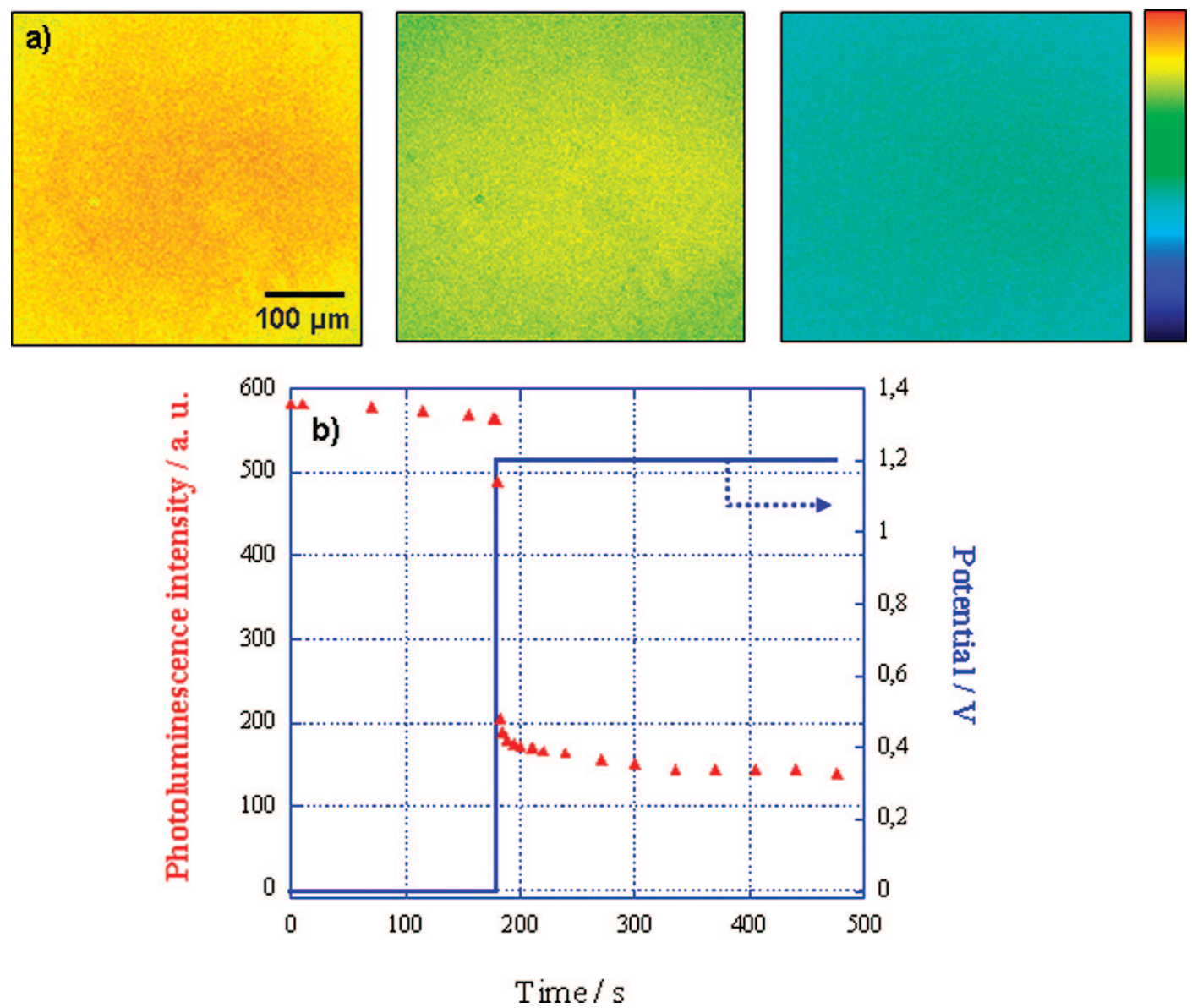

嵒

Figure 7. (a) Sequential photoluminescence images collected at different times $(0,1$, and $3 \mathrm{~s}$, from left to right) after applying a potential step from 0 to 1.2 V/Ag at an ITO electrode modified by 5 LB-Nafion layers which were loaded with Ru(bpy) ${ }_{3}{ }^{2+}$. All the images were coded according to the same color scale (right). The electrode was immersed in a solution containing $0.1 \mathrm{M} \mathrm{KNO}_{3}$. (b) Temporal photoluminescence intensity profile (triangles) collected after the application of the oxidizing potential step (blue line).

generated. The number of LB layers increases from top to bottom for the first four lines, while the lowest line refers to a spincoated electrode. From the analysis of this series of images, it can be observed that, at a reducing potential $(0 \mathrm{~V})$, the intensity of the emission increases by increasing the number of layers. The photoluminescence appears quite homogeneously distributed over the region of interest for the LB samples, apart from a decrease at the corners of some images, caused by the focusing of the incident light on the center of the image. Some linear scratches are present in the 30 and 40 layers, and no (or little) luminescence is observed on them. These negligible areas (caused by handling) are not taken into account in the following discussion. For the spin-coated samples, the luminescence intensity is higher and more localized. Comparison between LB and spin-coated Nafion films shows indeed a more uniform luminescence in the LB samples.

The images on the right side of Figure 6 were recorded $1 \mathrm{~s}$ after application of $1.2 \mathrm{~V}$ to the LB-Nafion samples. At this potential, the complex is oxidized and the nonphotoluminescent form is generated in the film. For the LB samples, one can see that the photoluminescence decreases dramatically and is completely turned off after $1 \mathrm{~s}$. Only for thicker samples (30 and 40 LB-Nafion layers), a clustered light intensity remains important. On the contrary, for the spin-coated sample, a large area remains luminescent even $20 \mathrm{~s}$ after application of $1.2 \mathrm{~V}$ (Figure 6). This indicates a much slower efficiency of the turning off for the spin-coated Nafion films with respect to the LBNafion films.
Figure 7 details the typical behavior of a 5-layer LB-Nafion film. In this electrode, $\mathrm{Ru}(\mathrm{bpy})_{3}{ }^{2+}$ luminescence is distributed very homogeneously, so indicating the formation of a very regular Nafion coating. Photoluminescence images were acquired as a function of time before and after application of the potential step. The oxidizing potential induces the uniform decrease of the photoluminescence (middle and right images of Figure 7a). Such dynamic imaging allows one to visualize directly the electrochemical behavior of the films. Indeed, the simultaneous decrease of luminescence with space and time is due to the corresponding decrease of the local Ru(II) concentration. From a sequence of photoluminescence images, the variations of the light intensity were calculated in the same region of interest after a potential step was imposed at the electrode. The dynamic photoluminescence intensity profile is displayed in Figure $7 \mathrm{~b}$. The collected light intensity decreases dramatically to about $30 \%$ in the first few seconds, and then it continues to decrease more slowly over a $100 \mathrm{~s}$ period up to reaching a constant value, that is, around $20 \%$ of the initial photoluminescence value.

Interestingly, a similar trend is observed also from the chronoamperometric plot reported in Figure 8. Also in this case, after application of the oxidizing potential, the current decreases quickly in the first $40 \mathrm{~s}$ and tends to reach a steady value for $t$ $>100 \mathrm{~s}$. The reasons behind this behavior are presently under investigation; they could be related to a redox reaction of $\mathrm{Ru}(\mathrm{III})$ with a species present in solution (e.g., $\left.\mathrm{OH}^{-}\right)^{44}$ to regenerate, partially, photoluminescent and electroactive $\mathrm{Ru}(\mathrm{II})$. 
Table 1. Diffusion Coefficient Values of Ru(bpy $)_{3}{ }^{2+}$ in NLBE with Different Numbers of Layers

\begin{tabular}{ccccccc}
\hline no. of layers & $v\left(\mathrm{~V} \mathrm{~s}^{-1}\right)$ & \multicolumn{1}{c}{$\phi(\mathrm{nm})^{c}$} & $m$ (no. of moles) & $S\left(\mathrm{~A}\left(\mathrm{~V} \mathrm{~s}^{-1}\right)^{-1 / 2}\right)$ & $D_{\text {app }}\left(\mathrm{cm}^{2} \mathrm{~s}^{-1}\right)^{a}$ & $D_{\text {app }}\left(\mathrm{cm}^{2} \mathrm{~s}^{-1}\right)^{b}$ \\
\hline 1 & 0.200 & $2.3 \pm 0.5$ & $(4.0 \pm 0.5) \times 10^{-11}$ & $(5.5 \pm 0.5) \times 10^{-5}$ & $(0.4 \pm 0.2) \times 10^{-12}$ & $(1.3 \pm 0.3) \times 10^{-12}$ \\
3 & 0.100 & $6.0 \pm 1.0$ & $(1.4 \pm 0.1) \times 10^{-10}$ & $(8.8 \pm 0.3) \times 10^{-5}$ & $(1.9 \pm 0.4) \times 10^{-12}$ & $(2.0 \pm 0.4) \times 10^{-12}$ \\
9 & 0.050 & $19.1 \pm 3.0$ & $(5.1 \pm 0.2) \times 10^{-10}$ & $(2.0 \pm 0.04) \times 10^{-4}$ & $(7.1 \pm 1.3) \times 10^{-12}$ & $(7.7 \pm 1.4) \times 10^{-12}$
\end{tabular}

${ }^{a}$ Calculated by using eq $2 .{ }^{b}$ Calculated by using eq $3{ }^{c}$ Average thicknesses measured by AFM as described in ref 20.

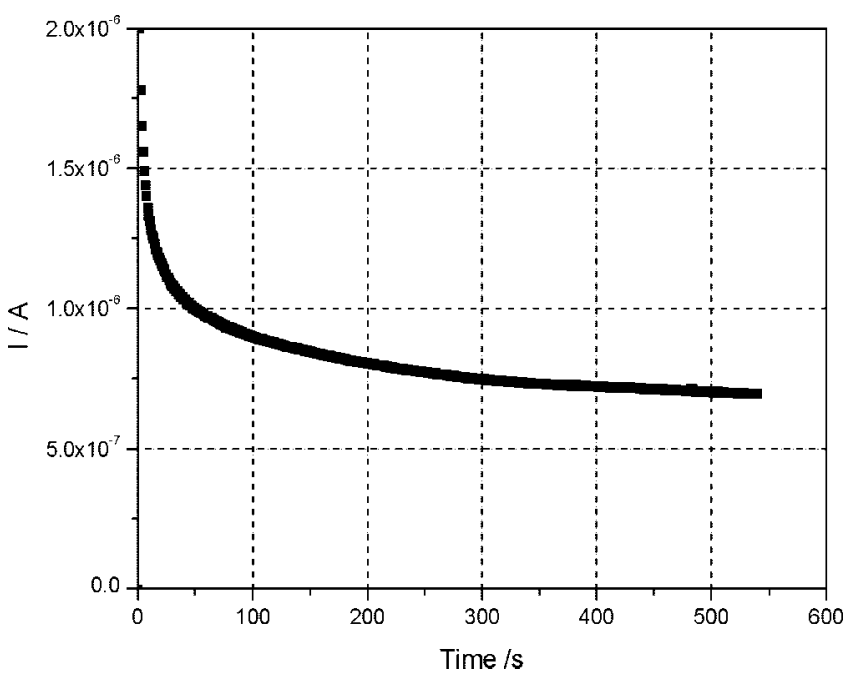

Figure 8. Chronoamperometric curve recorded at a 5-layer NLBE after application of a potential of $1.2 \mathrm{~V}$ to the electrode in pure supporting electrolyte solution (0.01 M PBS) after loading the electrode in $1 \mathrm{mM}$ $\mathrm{Ru}(\mathrm{bpy})_{3}{ }^{2+}$.

From the epifluorescence images at reducing potential, the average photoluminescence intensity was calculated for each sample. Figure 9 compares the photoluminescence intensity on the number of layers (Figure 9a) and the oxidation peak current recorded at the same electrodes (Figure 9b). By increasing the number of Nafion-LB layers, the number of redox photoluminescent centers increases proportionally, so that photoluminescence increases as well. The increase in photoluminescence scales roughly linearly with the number of LB-Nafion layers (Figure 9a). As shown in Figure 5b, also the amount of $\mathrm{Ru}(\mathrm{bpy})_{3}{ }^{2+}$ incorporated in the NLBE scales linearly with the number of layers, so indicating that the successive LB-Nafion layers are efficiently deposited over each other. The slight deviation from perfect linearity may be related to an inner-filter effect due to the absorbance of the sample; this effect is well-known in fluorescence techniques. ${ }^{45-47}$ It may be also related to selfquenching of the photoluminescence by the high concentration of $\mathrm{Ru}(\mathrm{bpy})_{3}{ }^{2+}$ centers within the compact LB-Nafion film. ${ }^{22}$ Regardless, the results confirm the efficiency of the LB techniques to accurately build multistructures of ultrathin Nafion films. The relation between the photoluminescence intensity and the oxidation peak current for different numbers of LB-Nafion layers is further illustrated using an adequate representation of the normalized data in Figure $7 \mathrm{~b}$. In this figure, peak currents and photoluminescence data are normalized with respect to the maximum values of the peak current and of the luminescence intensity, respectively. The electrochemical and photoluminescent data follow the same trend and scale quasi-linearly, with a slope close to unity. This means that the same fraction of immobilized

(43) White, H. S.; Leddy, J.; Bard, A. J. J. Am. Chem. Soc. 1982, 104, 48114817.

(44) Creutz, C.; Sutin, N. Proc. Natl. Acad. Sci. U.S.A. 1975, 72, 2858-2862.

(45) Leese, R. A.; Wehry, E. L. Anal. Chem. 1978, 50, 1193-1197.

(46) Yuan, P.; Walt, D. R. Anal. Chem. 1987, 59, 2391-2394.

(47) Tohda, K.; Lu, H.; Umezawa, Y.; Gratzl, M. Anal. Chem. 2001, 73, 2070-2077.

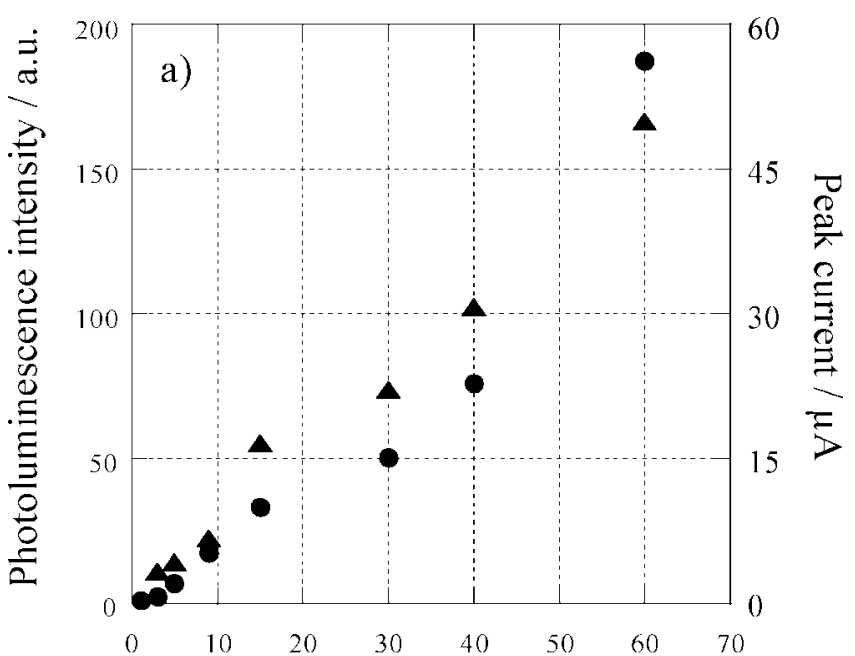

Number of layers

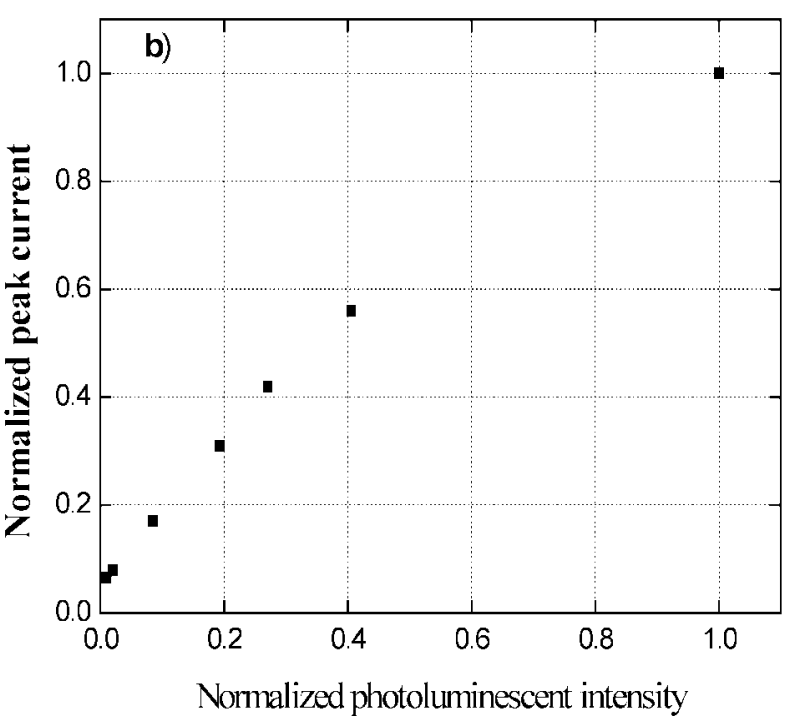

Figure 9. (a) Evolution of the normalized photoluminescence light intensity $(\boldsymbol{\Lambda})$ and of the oxidizing peak current $(\mathbf{O})$ for different numbers of LB-Nafion layers loaded with $\mathrm{Ru}(\mathrm{bpy}){ }_{3}{ }^{2+}$. (b) Correlation between the normalized photoluminescent intensity and the normalized peak current.

$\mathrm{Ru}(\mathrm{bpy})_{3}{ }^{2+}$ is both electroactive and photoluminescent in each layer of the LB-Nafion film. In other words, the ruthenium complex which is immobilized in the outer layers (i.e., far from the electrode surface) remains as electroactive as the complex incorporated closer to the electrode surface, so that electroactivity and photoluminescence of the immobilized $\mathrm{Ru}(\mathrm{bpy}){ }_{3}{ }^{2+}$ complex are invariant with film thickness. This indicates that electron hopping, which is the main transport mechanism for the case of $\mathrm{Ru}(\mathrm{bpy}) 3^{2+}$ in Nafion, ${ }^{43}$ allows an efficient charge transfer through all the complexes incorporated in the film.

As far as the oxidative switching off of luminescence is concerned, the faster response time of Nafion-LB versus spincoated Nafion films requires some further evaluation. By taking 
into account the Einstein-Smoluckowski equation for the diffusion length

$$
x=(2 D t)^{1 / 2}
$$

one can easily calculate the time required for switching off a film of thickness $\phi$ by

$$
t=\phi^{2} / 4 D
$$

For a 3-layer LB-Nafion film, the thickness is $6.0 \mathrm{~nm}(6.0 \times$ $\left.10^{-7} \mathrm{~cm}\right)$ with $D_{\text {app }}=2.0 \times 10^{-12} \mathrm{~cm}^{2} \mathrm{~s}^{-1}$ and $t_{\text {switch off }}=0.045$ s. A Nafion spin-coated film is much thicker, for example, 300 $\mathrm{nm}\left(3.0 \times 10^{-5} \mathrm{~cm}\right)$, and $D_{\text {app }}$ is larger, for example, $5 \times 10^{-10}$ $\mathrm{cm}^{2} \mathrm{~s}^{-1},{ }^{41}$ so that $t_{\text {switch off }}=0.45 \mathrm{~s}$, that is, approximately 1 order of magnitude larger.

All of this evidence indicates that lowering of the film thickness down to the nanometric level is very effective in shortening the switching time, notwithstanding the lowering of $D_{\text {app }}$ values in LB films. Moreover, in LB films, photoluminescence is turned off more quickly and more efficiently the lower the number of layers.

\section{Conclusions}

The redox probe $\mathrm{Ru}(\mathrm{bpy}){ }_{3}{ }^{2+}$ can be incorporated in Nafion-LB films while retaining its typical electrochemical and photochemi- cal behavior. The high compactness of Nafion-LB films reflects in very low values of apparent diffusion coefficients, which are in the order of $10^{-11}-10^{-12} \mathrm{~cm}^{2} \mathrm{~s}^{-1}$. Epifluorescence imaging of $\mathrm{Ru}(\mathrm{bpy}) 3^{2+}$ incorporated in the LB-Nafion films demonstrated to be a useful tool for obtaining information on the characteristics of the film. In particular, it was shown that the probe is quite homogeneously distributed in the film. Electroactivity and photoluminescence increases side by side by increasing the number of LB layers. Photoluminescence can be electrochemically turned off by applying an oxidizing potential. The response time to such a switching off is very fast for LB films, so indicating that the decrease of the film thickness down to the nanometer size prevails with respect to the opposite effect expected on the basis of the decrease in $D_{\text {app }}$ values which characterizes Nafion-LB films. This can be important when considering the use of LBcoated electrodes as detectors, for example, in flowing systems, where the response time is a crucial parameter.

Acknowledgment. We thank Caterina Pozzobon for some preliminary experiments and Prof. Giancarlo Battaglin (University of Venice) for profilometric measurements. Financial support by MUR (Rome, PRIN 2006) is gratefully acknowledged.

LA703998E 